\title{
Aphid-deprivation from Brassica plants results in increased isothiocyanate release and parasitoid attraction
}

\author{
Adriana J. Najar-Rodriguez ${ }^{1,2} \cdot$ Michael Friedli $^{1} \cdot$ Jeannine Klaiber $^{1} \cdot$ Silvia Dorn $^{1}$
}

Received: 20 April 2015/ Accepted: 22 July 2015 / Published online: 31 July 2015

(C) Springer Basel 2015

\begin{abstract}
In response to insect herbivory, plants release volatile blends that differ quantitatively, and in many cases also qualitatively, from those of undamaged plants. These altered blends can attract the herbivore's natural antagonists, and such herbivore-induced volatile blends have often been interpreted as co-evolved plant-insect antagonist signals. When comparing volatile profiles of infested Brassica oleracea var. gemmifera L. plants (Brassicales: Brassicaceae), on which Brevicoryne brassicae L. (Hemiptera: Aphididae) aphids are feeding, with previously infested plants (i.e., deprived of aphids four hours prior to volatile collection), we found that the emission of a particular volatile compound, the glucosinolate breakdown product allyl isothiocyanate, was significantly higher in the latter. We used dual choice olfactometry to evaluate attractiveness of plants and aphids to Diaeretiella rapae (M'Intosh 1855) (Hymenoptera: Braconidae), a parasitoid of $B$. brassicae. Previously infested plants deprived of the herbivore attracted significantly more parasitoids than infested plants, although aphid odours per se proved to be attractive. Mechanical damage approximating aphid stylet insertion remained without effect on emission of allyl isothiocyanate and on parasitoid response. The unexpected higher parasitoid attraction to previously infested than
\end{abstract}

Handling Editor: Michael Heethoff.

Adriana J. Najar-Rodriguez

adriana.najar-rodriguez@plantandfood.co.nz

1 Institute of Agricultural Sciences/Applied Entomology, ETH Zurich, Schmelzbergstrasse 9/LFO, 8092 Zurich, Switzerland

2 Present Address: The New Zealand Institute for Plant and Food Research Limited, Private Bag 11600 ,

Palmerston North, New Zealand infested plants is discussed here from the perspective of the plant and the parasitoid, as well as from the herbivore. Results suggest that while the host-finding mechanism of D. rapae has not evolved to allow this parasitoid a discrimination between herbivore-infested and previously infested plants, the herbivore seems able to suppress emission of a key volatile, thereby reducing its own olfactory detectability to its specialised natural antagonist.

Keywords Odour discrimination - Aphid · Wasp · Plant volatile $\cdot$ Glucosinolate Isothiocyanate

\section{Introduction}

In response to insect herbivory, plants release volatile blends that differ quantitatively and in many cases also qualitatively from those of undamaged plants (Kessler and Heil 2011; Mattiacci et al. 2001). These altered blends attract the herbivores' natural antagonists (Bernasconi Ockroy et al. 2001; Scascighini et al. 2005; Walling 2008; Zhang et al. 2013). Such volatiles have often been interpreted as co-evolved plant-antagonist signals (e.g., Dicke 1999; Turlings and Wäckers 2004), providing plants with a potent indirect defence mechanism (Dicke et al. 2003; Schuman et al. 2012). However, the defensive function of these volatiles and their contribution to plant fitness remains a topic of debate (Allison and Hare 2009; Kessler and Heil 2011).

In many plant-herbivore systems, volatile emissions change in the course of herbivore feeding. Remarkable temporal dynamics were found in emission of compounds both after the initiation of herbivory (Hoballah and Turlings 2005; Mattiacci et al. 2001; Scascighini et al. 2005) and after its discontinuation (Loughrin et al. 1994; 
Mattiacci et al. 2001; Kugimiya et al. 2010). Parasitoids in search of a suitable herbivore host are expected to respond to these dynamic changes in volatile emission and to possess the ability to discriminate between herbivore-infested plants and previously infested plants deprived of the herbivore. Specialist parasitoids, in particular, are expected to have a very sensitive and narrowly-tuned olfactory system for detection of chemical cues related to the specific herbivore-plant system (Ngumbi et al. 2009 and references therein).

Here, we tested the hypothesis that specialist parasitoids possess the ability to discriminate between herbivore-infested plants and previously infested plants deprived of the herbivore. We analysed volatiles emitted from the shoots of Brassica plants (Brassica oleraceae var. gemmifera) upon herbivory, with the herbivore still feeding or shortly after its removal, and tested the behavioural effects of these volatiles on the parasitoid Diaeretiella rapae. This wasp predominantly specialises in the parasitism of Brassicafeeding aphids (Pike et al. 1999; Girling et al. 2006; Blande et al. 2007) such as the cabbage aphid Brevicoryne brassicae. This insect herbivore uses mainly plants from the family Brassicaceae as a food source (Blackman and Eastop 2000). Brassica plants possess a well-studied chemical defence, the glucosinolate-myrosinase system (Hopkins et al. 2009). Glucosinolates and their hydrolytic enzymes, myrosinases, are stored in separate cellular compartments. Upon herbivore feeding, myrosinases get access to their glucosinolate substrates and glucosinolate hydrolysis results in the formation of toxic isothiocyanates and other biologically active volatiles (Winde and Wittstock 2011). Nevertheless, several specialised insect herbivores, including the aphid B. brassicae, use Brassica species as preferred food plants (Ahuja et al. 2010; Gutbrodt et al. 2012). These insects share the ability to tolerate or detoxify glucosinolates and/or their by-products (Hopkins et al. 2009). Specifically, they ensure that glucosinolate hydrolysis to isothiocyanates is avoided. Known mechanisms include avoidance of cell disruption, absorption of intact glucosinolates, conversion of glucosinolates to harmless compounds that are not substrates for myrosinases, and diversion of plant myrosinase-catalysed glucosinolate hydrolysis (Winde and Wittstock 2011). Interestingly, parasitoids of specialised Brassica-herbivores are attracted to volatile blends emanating from infested Brassica plants containing breakdown products from the glucosinolate-myrosinase system (Wittstock et al. 2003; Klaiber et al. 2013a), apparently because these are widespread across Brassica plants and phylogenetically conserved compared to other volatile groups (Dudareva et al. 2004).

Our objective was a quantified comparison of the volatile profiles of infested plants on which the specialised aphid was feeding, with those of plants from which this herbivore had been removed few hours prior to experiments (i.e., previously infested plants), and of the specialist parasitoid's preference for either olfactory choice. Furthermore, we wanted to compare the volatiles from these two groups of aphid-damaged plants to those emitted from mechanically damaged and undamaged plants, as well as their behavioural effects on the parasitoid.

\section{Materials and methods}

\section{Insects and plants}

Stock colonies of the aphid Brevicoryne brassicae and its parasitoid Diaeretiella rapae originating from field-collected insects in the region of Zurich, Switzerland, were maintained in the insectary under controlled conditions for about 60 generations. Aphids were kept on 4-week-old Brussels sprout plants (Brassica oleracea var. gemmifera). These plants, hereafter referred to as Brassica plants, were placed inside insect rearing cages $(30 \times 30 \times 30 \mathrm{~cm})$ (BugDorm, Megaview Science CO., Ltd, Taichung, Taiwan) and maintained under controlled conditions of $24{ }^{\circ} \mathrm{C}$ during the day and $18{ }^{\circ} \mathrm{C}$ at night, $50 \% \mathrm{RH}$ and a $16: 8 \mathrm{~h}$ L:D regime. Diaeretiella rapae parasitoids were maintained on B. brassicae aphids feeding on the Brassica plants in separate cages under the same conditions (Klaiber et al. 2013a). The Brassica plants used in all experiments were grown in commercial soil 'Klasman Substrat 2' (Klasmann-Deilmann GmbH, Geeste, Germany; a peat substitute with $\mathrm{pH}$-value 5.5, NPK: $280,320,360 \mathrm{mg} \mathrm{L}^{-1}$, $100 \mathrm{mg} \mathrm{L}^{-1}$ magnesium, trace elements and chelates) and were watered twice a week. Plants were 5-6 weeks old at the time of volatile collection and bioassays.

\section{Preparation of plant-host complexes for volatile collection and bioassays}

Aphid-infested Brassica plants (kept in BugDorm cages) were obtained by placing $500 \mathrm{~B}$. brassicae aphids of mixed ages $(N=30$ plants $)$ spread over two leaves per plant 1 week prior to experiments $(N=$ approximately 2000 aphids at the time of the experiments; $N$ determined by visual counting). Previously infested plants were treated the same way as the aphid-infested plants, but all aphids were carefully and gently removed with a paintbrush from the plants 4 hours prior to experiments. Honeydew was still visible on the leaves from which aphids were removed. However, we did not find any indication that the honeydew had any significant olfactory effect on the parasitoid (data not shown). To approximate mechanical damage inflicted by aphids while feeding on a plant, we relied on a method 
developed and used by Du et al. (1996) and Girling et al. (2006): three days prior to experiments, 4-5 leaves per plant were pierced with ca. 500 metal pins $(0.1 \mathrm{~mm}$ diameter, $12 \mathrm{~mm}$ length, Bioform Entomology \& Equipment, Nürnberg, Germany), and two days prior to experiments with an additional ca. 250 metal pins. All 750 pins were removed $4 \mathrm{~h}$ before the experiment.

\section{Volatile collection and chemical analyses}

Headspace volatiles from the differently treated and from undamaged plants ( $N=10$ plants each) were collected for $5 \mathrm{~h}$, from 12:00 (i.e., $6 \mathrm{~h}$ after onset of light) to 17:00, with a radial diffusive sampling system (Radiello model 120-2, Supelco, Buchs, Switzerland) (Vallat and Dorn 2005; Najar-Rodriguez et al. 2013). Volatile analysis followed the method described in Klaiber et al. (2013b).

Prior to volatile collection, sorbent cartridges $(60 \mathrm{~mm}$ long, $4.8 \mathrm{~mm}$ diameter) containing Tenax TA (250 $\pm 10 \mathrm{mg}$; particle size 20-35) were conditioned at $320{ }^{\circ} \mathrm{C}$ with a helium (5.0) flow of $60 \mathrm{~mL} \mathrm{~min}^{-1}$ for $7 \mathrm{~h}$. For storage, the cartridges were enclosed in stainless steel tubes that were sealed with brass Swagelok caps (Arbor Ventil \& Fitting AG, Niederrohrdorf, Switzerland). Volatile samples were taken from the aboveground part of an entire plant that was, together with a sorbent cartridge inserted in a diffusive body, enclosed in a polyester bag (Toppits Brat-Schlauch, Melitta GmbH, Egerkingen, Switzerland) preconditioned at $120{ }^{\circ} \mathrm{C}$ for $12 \mathrm{~h}$. Samples of volatiles from an empty polyester bag and from the surrounding air were collected as controls ( $N=10$ plants).

Analysis by gas chromatography-mass spectrometry (GC-MS) was used to elucidate the qualitative and quantitative composition of the headspace volatiles. The volatiles were desorbed from the sorbent tubes with a Unity/Ultra thermal desorption (TD) system (Markes Int. Ltd, Llantrisant, UK) interfaced with a Hewlett-Packard 6890 GC5976 MS (Hewlett-Packard Co., Palo Alto, CA, USA). The TD flow path was purged for $7 \mathrm{~min}$ prior to tube desorption. The tube was then desorbed for $12 \mathrm{~min}$ at $280{ }^{\circ} \mathrm{C}$ and refocused on a cold trap (Tenax TA/Carbograph 1TD) at $-10{ }^{\circ} \mathrm{C}$. The trap was desorbed for $7 \mathrm{~min}$ at $300{ }^{\circ} \mathrm{C}$ [carrier gas: helium (10 psi); transfer line temperature: $\left.160{ }^{\circ} \mathrm{C}\right]$. The GC was equipped with a retention gap (uncoated; $5 \mathrm{~m} \times 0.25 \mathrm{~mm}$; Hewlett-Packard Co., Palo Alto, CA, USA) and a DB-5 ms column (30 m $\times 0.25 \mathrm{~mm}$; $0.25 \mu \mathrm{m}$ film thickness; J\&W Scientific, Folsom, CA, USA). The GC oven temperature was held for $5 \mathrm{~min}$ at $40^{\circ} \mathrm{C}$, then increased to $100{ }^{\circ} \mathrm{C}$ at $3{ }^{\circ} \mathrm{C} / \mathrm{min}$, up to $320{ }^{\circ} \mathrm{C}$ at $5{ }^{\circ} \mathrm{C} / \mathrm{min}$, and held at $320{ }^{\circ} \mathrm{C}$ for $4 \mathrm{~min}$ (transfer line temperature: $280{ }^{\circ} \mathrm{C}$; ionization energy: $70 \mathrm{eV}$; ion source temperature: $230{ }^{\circ} \mathrm{C}$; quadrupole temperature: $150{ }^{\circ} \mathrm{C}$; mass range: $30-500 \mathrm{amu}$ ). ChemStation software (MSD Productivity Chem Station software, Agilent Technologies Inc., Santa Clara, CA, USA) was used for volatile identification and quantification. Identification was initially performed via comparison of recorded spectra to spectra published in the NIST library and in Adams (1995). Linear retention indices (RIs) were calculated using a mixture of $n$-alkanes $\left(\mathrm{C}_{7}-\mathrm{C}_{40}\right)$ (Supelco, Bellefonte, PA, USA) as standards and were compared with those in Adams (1995). Identification of all compounds was confirmed by GC/MS co-injection of synthetic compounds.

For semiquantification, cyclohexane in the gas phase was injected onto the sorbent cartridge as internal standard prior to GC-MS analysis. The internal standard was prepared as in Piskorski and Dorn (2010), based on the method described in Dewulf et al. (1999), and the Henry's law coefficient for calculation was used from therein. Cyclohexane $(0.235 \mathrm{mmol} ; 25.5 \mu \mathrm{L})(\geq 99.8 \%$, Sigma-Aldrich, Buchs, Switzerland) was first dissolved in methanol $(100 \mathrm{~mL})$. Aliquots of the cyclohexane/methanol solution $(50 \mu \mathrm{L})$ were then dissolved in Milli-Q water $(4.0 \mathrm{~mL})$ in a series of $24.5 \mathrm{~mL}$ amber glass vials which were tightly closed with Mininert valves (Sigma-Aldrich, Buchs, Switzerland) and finally incubated in a thermostatic chamber (Heraeus BK 6160 testing chamber, Thermo Fisher Scientific, Schwerte, Germany) at $25.0 \pm 0.1{ }^{\circ} \mathrm{C}$ for $24 \mathrm{~h}$. Prior to desorption, aliquots of the gas phase $(2.0 \mathrm{~mL})$ that were taken from consecutive vials were injected into the sorbent tubes with a gastight syringe with a He flow of $60 \mathrm{~mL} \mathrm{~min}^{-1}$.

\section{Olfactometer bioassays}

The olfactometer consisted of a Y-shaped glass tube $(2.5 \mathrm{~cm}$ diameter, $24 \mathrm{~cm}$ biforked arm length and $22 \mathrm{~cm}$ common arm length) connected to two tubular glass tubes $(5 \mathrm{~cm}$ diameter and $30 \mathrm{~cm}$ length). Each of these tubes was, in turn, connected to a small glass chamber $(5 \mathrm{~cm}$ diameter, $14.5 \mathrm{~cm}$ length) provided with a glass frit that prevented the parasitoids from going further into the direction of the odour source, while still allowing the passing of airflow. These small glass chambers were connected to larger glass chambers (15.9 $\mathrm{cm}$ diameter, $40 \mathrm{~cm}$ height) in which the plants were placed. Moistened, charcoal-filtered air was drawn into each of the two chambers and into the Y-tube arms at a rate of $450 \pm 10 \mathrm{~mL} / \mathrm{min}$. Airflow rates were always calibrated before and during experiments with an electronic flow meter (Agilent flow meter ADM 1000; Agilent Technologies, Centerville, DE, USA).

Bioassays were conducted from 11:00 (i.e., $5 \mathrm{~h}$ after onset of light) to $16: 00$ at $24.9 \pm 0.3{ }^{\circ} \mathrm{C}$ and $54.7 \pm 1.3 \%$ $\mathrm{RH}$. This period in the diel rhythm of the wasps corresponds to the time window when adult female wasps show increased locomotor activity (M. Friedli and A. NajarRodriguez, personal observations). Providing parasitoids 
with a positive experience that is associated with an odour can facilitate the effective use of volatile chemicals by parasitoids, or enhance their ability to respond to these odours, thus increasing foraging efficiency (Grasswitz and Paine 1993; Micha et al. 2000). Thus, newly emerged female wasps were exposed to Brassica leaves infested with $B$. brassicae aphids upon emergence. One- to 2-dayold single females were chosen at random and were transferred to the bioassay room $30 \mathrm{~min}$ prior to the bioassays. Each female wasp was then released at the entrance of the common arm of the Y-tube and exposed to a particular choice of odours emanating from the differently treated Brassica plants ( $N=30$ wasps/odour choice). Choices provided consisted of an undamaged plant versus: (a) a plant infested with or (b) a plant previously infested with approximately 2000 B. brassicae aphids, (c) a mechanically damaged plant approximating wounding by aphids, or (d) clean air. Wasps were also presented with an olfactory choice between an aphid-infested and a previously infested plant. A choice between an undamaged plant and clean air served as a control. To exclude the influence of soil-borne volatiles, plant pots were always wrapped tightly with a polyester bag (Toppits Brat-Schlauch, Melitta GmbH, Egerkingen, Switzerland) preconditioned at $150{ }^{\circ} \mathrm{C}$ overnight. To test for an olfactory preference between aphids alone (ca. 2000) and clean air, the tubular glass chamber and the adjoined small glass chamber were replaced by a clean and smaller tubular glass chamber $(2.8 \mathrm{~cm}$ diameter, $30 \mathrm{~cm}$ length), provided with a glass frit to prevent the living aphids from crawling in the direction of the air flow and the female wasps from visual contact with the hosts. For each odour choice tested, a new set of plants (or aphids) was used, and each wasp was tested only once.

The response of each female wasp to the different odour choices was observed for a maximum of $20 \mathrm{~min}$. A choice was considered to have been made if a female entered one of the biforked arms and then crossed a score line drawn $3 \mathrm{~cm}$ from the intersection of the tube (Girling et al. 2006). By contrast, a choice was considered to have not been made if the female remained in the common arm of the Y-tube throughout the observation period (Bertschy et al. 1997). Five fluorescent lamps (four OSRAM L 58 W/12 lamps and one OSRAM L 58 W/22 lamp, OSRAM GmbH, Munich, Germany) provided diffused light at an intensity of about 2150 lux. Visual cues for the wasps were minimized by surrounding the olfactometer with white paper walls while still allowing behavioural observations. New females were tested on each of three to five consecutive days to account for daily differences in wasp responsiveness, with each treatment been tested in a randomized order at each of these days. Between every female tested, the position of the Y-tube arms was swapped to avoid positional bias. After each day of bioassays, all olfactometer parts were rinsed with acetone and hexane, washed in a detergent solution in hot water, and finally, rinsed with distilled water. Then, all the cleaned parts were placed in an oven overnight at $120^{\circ} \mathrm{C}$.

\section{Statistical analysis}

To identify differences in the emission of volatile compounds between the different plant-host complexes tested, one-way ANOVAs were carried out for each individual compound. Whenever effects were detected, means were subsequently compared by the Tukey's HSD test. Prior to analysis, volatile data were $\log _{10}(\mathrm{X}+1)$ transformed to meet the assumptions of normality and heteroscedasticity for parametric tests. Principal component analysis (PCA) was applied to yield a 2D display of the multivariable set of data in order to search for similarities/differences in samples of headspace volatiles (JMP 8.0, SAS Institute Inc., Cary, NC, USA). Results of the Y-tube olfactometer bioassays were analysed for preference (i.e., percentage of females that made a choice between the two odours tested) with a binomial test of goodness-of-fit with the null hypothesis of no preference for one odour in a particular odour combination. Females that did not make a choice and did not move more than half way up the common arm of the olfactometer, i.e., $10 \mathrm{~cm}$, within the first $5 \mathrm{~min}$, were excluded from the analyses. Thus, for each binomial test the number of females considered for analysis varied accordingly. Female responsiveness (i.e., proportion of females that made a choice, regardless of that choice) across the different odour combinations was then analysed using a binomial generalized linear model. Differences between pairs of odour combinations were determined using the standard errors of differences of predictions to calculate $t$ - and $P$ values. All analyses were conducted using Genstat 14th edition (VSN International Ltd, Hemel Hempstead, UK).

\section{Results}

\section{Chemical analyses}

Total amounts of emitted volatiles from aphid-infested and previously infested plants did not differ significantly from one another $(F=0.005, d f=1, P=0.943)$. However, several qualitative differences, and one quantitative difference pertaining to a single compound, were noted between the volatile profiles emanating from these two treatments (Table 1). The principal component analysis clearly separated the aphid-infested and previously infested plants into two independent clusters (Fig. 1). Qualitative 
Table 1 Volatile compounds detected in the headspace of Brassica plants either infested or previously infested by Brevicoryne brassicae aphids, mechanically damaged or left undamaged

\begin{tabular}{|c|c|c|c|c|c|c|}
\hline Compound & RI & Infested & Previously infested & Mechanically damaged & Undamaged & $P$ \\
\hline \multicolumn{7}{|l|}{ Terpenes } \\
\hline$\alpha$-Thujene & 922 & $252.9 \pm 52.9 \mathrm{~b}$ & $241.5 \pm 97.5 b c$ & $455.55 \pm 84.3 \mathrm{a}$ & $138.1 \pm 21.1 \mathrm{c}$ & $<0.05$ \\
\hline$\alpha$-Pinene & 927 & $150.6 \pm 27.1 b$ & $157.5 \pm 64.9 b$ & $214.84 \pm 37.7 \mathrm{a}$ & $78.2 \pm 10.8 \mathrm{c}$ & $<0.05$ \\
\hline Sabinene & 967 & $467.6 \pm 104.7 b$ & $466.0 \pm 184.5 \mathrm{ab}$ & $779.75 \pm 147.7 \mathrm{a}$ & $222.3 \pm 35.6 \mathrm{c}$ & $<0.05$ \\
\hline$\beta$-Pinene & 971 & $71.3 \pm 14.5 b$ & $84.7 \pm 35.9 b$ & $462.33 \pm 73.5 \mathrm{a}$ & $30.0 \pm 3.9 \mathrm{c}$ & $<0.001$ \\
\hline Limonene & 1025 & $1170.4 \pm 206.8 \mathrm{a}$ & $912.0 \pm 293.0 \mathrm{a}$ & $201.66 \pm 43.2 \mathrm{c}$ & $387.8 \pm 31.9 b$ & $<0.001$ \\
\hline Eucalyptol & 1027 & $260.3 \pm 38.6 \mathrm{a}$ & $255.8 \pm 99.2 \mathrm{a}$ & $372.20 \pm 92.3 \mathrm{a}$ & $113.9 \pm 20.6 b$ & $<0.05$ \\
\hline \multicolumn{7}{|l|}{ Hydrocarbons } \\
\hline Undecene $^{\mathrm{A}}$ & 1092 & - & - & - & $72.5 \pm 9.0$ & NA \\
\hline Tetradecane & 1400 & - & - & $3008.17 \pm 756.4$ & - & NA \\
\hline \multicolumn{7}{|l|}{ Aldehydes } \\
\hline Undecanal & 1307 & $972.3 \pm 122.7$ & - & - & - & NA \\
\hline \multicolumn{7}{|l|}{ Ketone } \\
\hline 3-Pentanone & 694 & $61.78 \pm 13.2$ & - & - & - & NA \\
\hline \multicolumn{7}{|l|}{ Disulfide } \\
\hline Dimethyl disulfide & 742 & $189.3 \pm 49.8 \mathrm{a}$ & $223.20 \pm 106.9 a$ & - & $30.2 \pm 7.9 b$ & $<0.001$ \\
\hline \multicolumn{7}{|l|}{ Nitrogen compound } \\
\hline Allyl isothiocyanate & 877 & $45.4 \pm 36.4 b$ & $233.8 \pm 82.8 \mathrm{a}$ & - & - & $<0.001$ \\
\hline Total & & $3641.9 \pm 601.8 b$ & $2574.6 \pm 877.2 b$ & $5494.5 \pm 1159.8 \mathrm{a}$ & $1073.1 \pm 119.9 \mathrm{c}$ & $<0.001$ \\
\hline
\end{tabular}

Concentration (in ng) per plant and compound (mean $\pm \mathrm{SE}$ ). All compounds were definitely identified. Source of standards: Sigma-Aldrich (Buchs, Switzerland). $N=10$ plants/treatment. $P$ values based on one-way ANOVAs followed by Tukey's HSD test for each single compound. Values for each volatile compound followed by the same letter are not significantly different from one another

$R I$ retention index, $N A$ non-applicable

${ }^{A}$ Compound not detected in more than $60 \%$ of the samples

differences in the composition of the blends from these two groups of plants consisted of undecanal and 3-pentanone, which were detected exclusively from aphid-infested plants (Table 1). The quantitative difference between these two blends pertained to allyl isothiocyanate. Interestingly, its concentration was five times higher in the headspace of previously infested compared with aphid-infested plants $(F=11.05, d f=1, P<0.001)$. This nitrogen-containing compound was not detected in any of the blends emitted by the remaining groups of plants tested.

Volatile compounds leading to qualitative differences between aphid-damaged (i.e., infested and previously infested) plants and undamaged plants comprised, besides of allyl isothiocyanate, only undecene, which was solely detected from undamaged plants (Table 1). Volatile compounds leading to quantitative differences among aphiddamaged and undamaged plants comprised most terpenes and dimethyl disulfide. Both total amounts of terpenes and dimethyl sulfide were detected at significantly higher concentrations from both aphid-infested and previously infested plants than undamaged plants $(F=40.92, d f=2$, $P<0.001$ and $F=9.33, d f=2, P<0.001$, respectively; Table 1).
Compositions of volatile blends from mechanically damaged plants differed quantitatively, and to some extent qualitatively from those emitted by aphid-damaged and undamaged plants (Table 1; Fig. 1). Quantitative differences in the composition of the blends from mechanically damaged plants consisted of increased emissions of several terpenes, whereby total terpene emission was statistically similar to that from aphid-damaged plants, but significantly higher than that from undamaged plants $(F=23.48$, $d f=2, P<0.001)$. Qualitatively, tetradecene was the only compound solely detected from mechanically damage plants, and due to its high concentration, total volatile amounts emitted by mechanically damaged plants significantly exceeded those detected from any other group of plants (Table 1).

\section{Behavioural bioassays}

Diaeretiella rapae female wasps significantly preferred volatiles from previously infested over aphid-infested plants $(P=0.049, N=17)$, whereas they still preferred volatiles from aphid-infested plants over those from undamaged plants $(P=0.007, N=15)$ (Fig. 2). When 
given a choice between odours from mechanically damaged and undamaged plants, all responding females preferred odours from mechanically damaged plants, but this difference missed the level of significance $(P=0.062$,

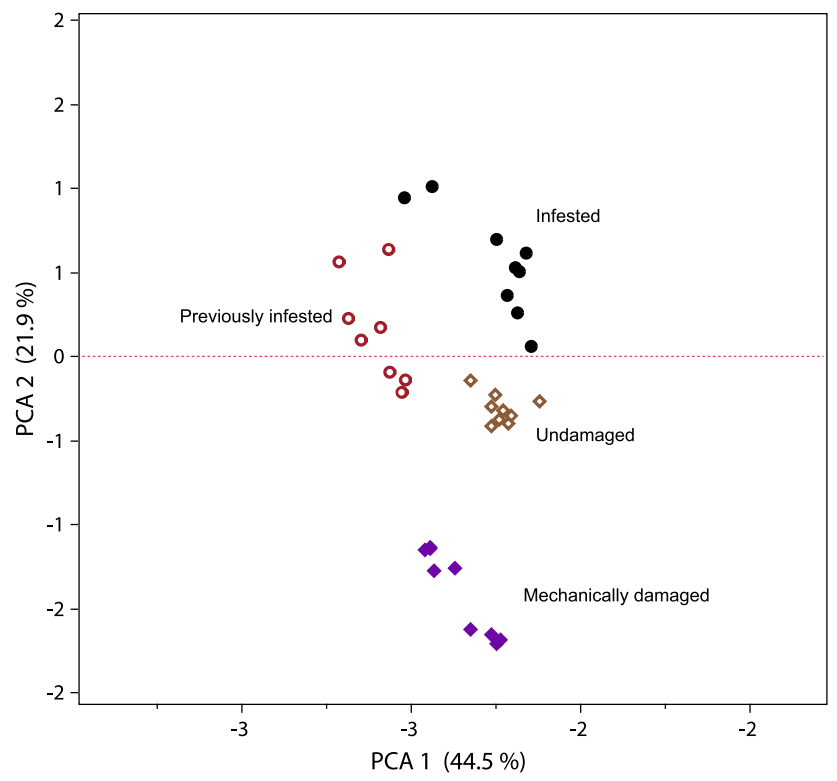

Fig. 1 Principal component analysis representation of volatiles detected in the headspace of different Brassica plant-host complexes. Plants were either infested or previously infested with Brevicoryne brassicae aphids, mechanically damaged in approximation to aphid wounding or left undamaged. $N=10$ plants/treatment. Contributions of principal components to separation of the samples are indicated on the axes
$N=5$ ). Furthermore, responsiveness, i.e., the percentage of females that made a choice, was two to three times lower in this choice experiment where the aphid host was absent, compared to the choices where both the aphid host and the plant were simultaneously present (i.e., aphid-infested vs undamaged plants, and aphid-infested vs previously infested plants; $t=5.33$ and 5.75, respectively; $P<0.001$ in both cases). When wasps were exposed to odours from the aphid host alone versus clean air, the wasps significantly preferred aphid odours $(P=0.002, N=14)$, with a high responsiveness not significantly different from that noted in choices where the aphid host and the plant were simultaneously present (i.e., aphid-infested vs undamaged plants, and aphid-infested vs previously infested plants; $t=0.35$ and $0.22 ; P=0.365$ and 0.414 , respectively) (Fig. 2).

Finally, wasps did not prefer volatiles from undamaged plants over clean air (control) $(P=0.625, N=4)$, and responsiveness was again significantly lower than in any choice involving aphids (aphid-infested vs undamaged plants, aphid-infested vs previously infested plants, and aphids alone vs clean air; $t=4.18,4.46$ and 4.59, respectively; $P<0.001$ in all cases) (Fig. 2).

\section{Discussion}

In many host plant-herbivore systems, herbivore-induced volatile emissions continue after the herbivore has abandoned the plant (Mattiacci et al. 2001; Kugimiya et al. 2010). Thus, for host detection by parasitoids to be

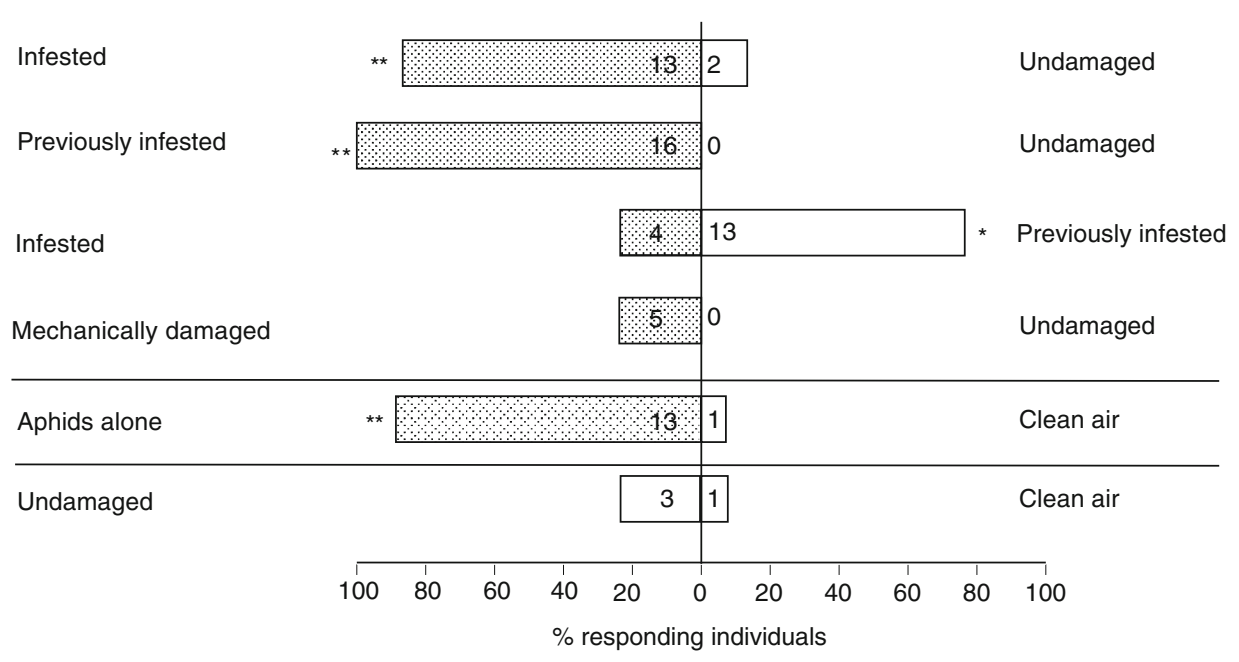

Fig. 2 Behavioural responses of experienced Diaeretiella rapae female wasps in Y-tube olfactometer bioassays. Dual choices involved odours from Brassica plants infested with or previously infested with the host, the aphid Brevicoryne brassicae, or mechanically damaged in approximation to aphid wounding. Females were also exposed to odours emitted by aphids alone vs clean air. The control was the choice between undamaged plants and clean air.
$N=30$ females/odour combination tested. The bars represent the percentage of tested parasitoids that made a particular choice, and the actual number of females making a choice for either of the two odours offered is given within the corresponding bar. For each odour combination, a binominal test of goodness-of-fit was made to compare the percentage of females that made a choice between the two odours tested. $* P<0.05, * * P<0.01$ 
effective, these natural antagonists are expected to discriminate between herbivore-infested plants and previously infested plants deprived of the herbivore in favour of the still infested plants. Here we tested this hypothesis by comparing volatile profiles of infested Brassica oleracea var. gemmifera plants, on which Brevicoryne brassicae aphids are feeding, with previously infested Brassica plants (i.e., deprived of aphids four hours prior to volatile collection). We also tested the attractiveness of such plants to the aphid parasitoid Diaeretiella rapae in a Y-tube olfactometer set up. We found that the emission of a particular volatile compound, the glucosinolate breakdown product allyl isothiocyanate, was significantly higher in previously infested plants compared to aphid-infested plants. Interestingly, such previously infested plants deprived of the herbivore for four hours attracted significantly more $D$. rapae wasps than the infested plants. Mechanical damage approximating aphid stylet insertion remained without effect on emission of allyl isothiocyanate and on parasitoid response. The unexpected higher attraction of a parasitoid to previously infested than infested plants is a novel result not yet reported in this or in other systems. This remarkable finding needs to be considered from the perspective of the plant and the parasitoid (Mattiacci et al. 2001), as well as from the perspective of the herbivore (Walling 2008).

Both aphid-infested and previously infested plants had suffered mechanical damage by stylet insertion, and to approximate this mechanical effect, we inserted pins into plants. In contrast to aphid-damaged plants, such plants damaged mechanically by artificial means did not release any allyl isothiocyanate, whereas the total amount of released terpenes reached a similar level in all these differently treated groups of plants. The finding that only few parasitoid wasps responded to odours from mechanically damaged plants in contrast to odours from aphid-damaged plants suggests that some olfactory information present only in the volatiles from aphid-damaged plants-such as isothiocyanate (Blande et al. 2007)_might have contributed decisively to the marked differences in the wasps' responsiveness.

A comparison of volatile profiles identified from aphidinfested and previously infested plants shows that two ubiquitous compounds reported from various Brassica plants (Bruinsma et al. 2009; Taveira et al. 2009; Mathur et al. 2013), the aldehyde undecanal and the ketone 3-pentanone, had disappeared in the latter. This is likely because the induction of plant volatile emission had been short-lived. A compound known specifically from herbivore-damaged plants of the Brassicales, the nitrogencontaining allyl isothiocyanate (Winde and Wittstock 2011), occurred in the blends emitted from previously infested and from aphid-infested plants, but was not detected in blends from undamaged or from mechanically damaged plants. Upon herbivory, Brassica plants release the enzyme myrosinase, which catalyses the hydrolysis of glucosinolates, yielding nitrogen-containing breakdown products such as allyl isothiocyanate (Kazana et al. 2007; Ahuja et al. 2010). Isothiocyanates appear to play a pivotal role in the attraction of $D$. rapae, a natural antagonist specialized to parasitize Brassica-feeding aphids. Using Y-tube olfactometry, D. rapae female wasps were significantly attracted to a synthetic isothiocyanate when offered versus a clean air (Blande et al. 2007), or to volatiles from a high-isothiocyanate producing Brassica-line in a choice trial with nearly isogenic, low-isothiocyanate producing line (Bradburne and Mithen 2000). The current study shows that this parasitoid was significantly more attracted to both aphid-infested and previously infested plants than to undamaged plants. Furthermore and counter-intuitively, attraction to previously infested plants was significantly stronger than to aphid-infested plants, with the previously infested plants (now devoid of the herbivore) emitting significantly higher amounts of allyl isothiocyanate than the plants still carrying the feeding aphids. This unexpected behaviour is striking given that volatiles from aphids alone were per se also attractive to the wasp. This attraction to the aphids was noted even though they were removed carefully from the plants. Thus, we cannot exclude that they released an alarm pheromone, which are known to attract D. rapae (Foster et al. 2005 and references therein) possibly even in conjunction with allyl isothiocyanate, as documented for disturbed $B$. brassicae (Kazana et al. 2007). Altogether, our results indicate that attraction of $D$. rapae wasps to allyl isothiocyanate may not be fine-tuned to the presence of the feeding aphid host, but rather to the emission of this volatile. Thus, it seems that the hostfinding mechanism of this parasitoid has not evolved to allow $D$. rapae to discriminate between herbivore-infested plants and induced plants deprived of the herbivore.

Insect herbivores have been typically perceived as mere inducers of plant defence responses, passively suffering parasitism or predation from the attracted natural antagonists. However, first indications emerging from recent work seem to contradict this view by demonstrating that insect herbivores might actively manipulate plant defence responses (Lawrence et al. 2008; Pareja et al. 2012; Schwartzberg et al. 2011). Helicoverpa zea and Spodoptera exigua caterpillars' saliva contain glucose oxidases that reduce induced defences (Bede et al. 2006; Musser et al. 2002). Attacks by Leptinotarsa decemlineata beetles suppress transcription of genes encoding for proteinase inhibitors also involved in plant defence (Lawrence et al. 2008). The silverleaf whitefly Bemisia tabaci biotype B prevents the activation of the jasmonic acid-regulated defences by Arabidopsis plants, which decreases plant defences and enhances nymphal performance (Walling 
2008; Zhang et al. 2013). Feeding by the specialist Lipaphis erysimi aphid was hypothesized to suppress floral volatile production by white mustard plants through modification of interrelated defence pathways and chemical signalling by introducing salivary factor(s) into the phloem (Pareja et al. 2012). Thus, the much higher amounts of allyl isothiocyanate released from previously infested plants deprived of the herbivore compared to infested plants could indicate a similar mechanism underlying our findings. Feeding by $B$. brassicae appears to induce the release of allyl isothiocyanate from Brassica plants, but its concentration is kept low as long the aphid is sucking, compared to when the plants are deprived of the aphid. Suppression of volatiles by the herbivore could hold the potential to act in its favour (Walling 2008), in this case allowing the aphid to circumvent plant defence by reducing its olfactory detectability to its specialised natural antagonist. Such potential mechanism, as postulated in general terms for phloem-sucking insects by Walling (2008), is compatible with the differing volatile profiles of aphidinfested versus previously infested Brassica plants, and the marked preference of D. rapae wasps for the latter in our study. Reducing the emission of herbivore-induced plant volatiles could be of major benefit to the aphid B. brassicae, as smaller volatile emissions-here five times less allyl isothiocyanate-could result in fewer indirect defences, in line with the theory by Walling outlined above (2008). Responses of natural antagonists to volatile suppression by their host aphid have scarcely been investigated so far. In the Brassica specialist aphid Lipaphis erysimi, suppression of floral volatiles did not alter the olfactory orientation of its generalist ladybird predator Coccinella septempunctata, and it also failed to outsmart its specialised parasitoid wasp D. rapae in its olfactory host location (Pareja et al. 2012). Further experiments are encouraged to investigate mechanisms underlying potential suppression of plant volatile emissions, and consequences in the tritrophic context used in this study and in further systems.

Acknowledgments We thank Brittany Hawkins-Orschel and Maya Senn for help with the experiments, Duncan Hedderley for statistical advice, and Kathrin Tschudi-Rein, Jana Collatz and two anonymous reviewers for useful comments on the manuscript.

\section{References}

Adams R (1995) Identification of essential oil components by gas chromatography/mass spectrometry. Allured Publishing Corporation, Illinois

Ahuja I, Rohloff J, Bones AM (2010) Defence mechanisms of Brassicaceae: implications for plant-insect interactions and potential for integrated pest management. A review. Agron Sustain Dev 30:311-348
Allison JD, Hare JD (2009) Learned and naive natural enemy responses and the interpretation of volatile organic compounds as cues or signals. New Phytol 184:768-782

Bede JC, Musser RO, Felton GW, Korth KL (2006) Caterpillar herbivory and salivary enzymes decrease transcript levels of Medicago truncatula genes encoding early enzymes in terpenoid biosynthesis. Plant Mol Biol 60:519-531

Bernasconi Ockroy ML, Turlings TJC, Edwards PJ, FritzscheHoballah ME, Ambrosetti L, Bassetti P, Dorn S (2001) Response of natural populations of predators and parasitoids to artificially induced volatile emissions in maize plants (Zea mays L.). Agric For Entomol 3:201-209

Bertschy C, Turlings TCJ, Bellotti AC, Dorn S (1997) Chemicallymediated attraction of three parasitoid species to mealybuginfested cassava leaves. Fla Entomol 80:383-395

Blackman RL, Eastop VF (2000) Aphids on the world's crops, an identification and information guide. The Natural History Museum, London

Blande J, Pickett J, Poppy G (2007) A comparison of semiochemically mediated interactions involving specialist and generalist Brassica-feeding aphids and the braconid parasitoid Diaeretiella rapae. J Chem Ecol 33:767-779

Bradburne RP, Mithen R (2000) Glucosinolate genetics and the attraction of the aphid parasitoid Diaeretiella rapae to Brassica. Proc R Soc Lond B Biol Sci 267:89-95

Bruinsma M, Posthumus MA, Mueller MJ, van Loon JJA, Dicke M (2009) Jasmonic acid-induced volatiles of Brassica oleracea attract parasitoids: effects of time and dose, and comparison with induction by herbivores. J Exp Bot 60:2575-2587

Dewulf J, Van Langenhove H, Everaert P (1999) Determination of Henry's law coefficients by combination of the equilibrium partitioning in closed systems and solid-phase microextraction techniques. J Chromatogr A 830:353-363

Dicke M (1999) Are herbivore-induced plant volatiles reliable indicators of herbivore identity to foraging carnivorous arthropods? Entomol Exp Appl 91:131-142

Dicke M, van Poecke RMP, de Boer JG (2003) Inducible indirect defence of plants: from mechanisms to ecological functions. Basic Appl Ecol 4:27-42

Du YJ, Poppy GM, Powell W (1996) Relative importance of semiochemicals from first and second trophic levels in host foraging behavior of Aphidius ervi. J Chem Ecol 22:15911605

Dudareva N, Pichersky E, Gershenzon J (2004) Biochemistry of plant volatiles. Plant Physiol 135:1893-1902

Foster SP, Denholm I, Thompson R, Poppy GM, Powell W (2005) Reduced response of insecticide-resistant aphids and attraction of parasitoids to aphid alarm pheromone; a potential fitness trade-off. Bull Ento Res 95:37-46

Girling R, Hassall M, Turner J, Poppy G (2006) Behavioral responses of the aphid parasitoid Diaeretiella rapae to volatiles from Arabidopsis thaliana induced by Myzus persicae. Entomol Exp Appl 120:1-9

Grasswitz TR, Paine TD (1993) Effect of experience on in-flight orientation to host-associated cues in the generalist parasitoid Lysiphlebus testaceipes. Entomol Exp Appl 68:219-229

Gutbrodt B, Dorn S, Unsicker SB, Mody K (2012) Species-specific responses of herbivores to within-plant and environmentally mediated between-plant variability in plant chemistry. Chemoecology 22:101-111

Hoballah ME, Turlings TCJ (2005) The role of fresh versus old leaf damage in the attraction of parasitic wasps to herbivore-induced maize volatiles. J Chem Ecol 31:2003-2018

Hopkins RJ, van Dam NM, van Loon JJA (2009) Role of glucosinolates in insect-plant relationships and multitrophic interactions. Annu Rev Entomol 54:57-83 
Kazana E, Pope TW, Tibbles L, Bridges M, Pickett JA, Bones AM, Powell G, Rossiter JT (2007) The cabbage aphid: a walking mustard oil bomb. Proc R Soc B 274:2271-2277

Kessler A, Heil M (2011) The multiple faces of indirect defences and their agents of natural selection. Funct Ecol 25:348-357

Klaiber J, Najar-Rodriguez AJ, Dialer E, Dorn S (2013a) Elevated carbon dioxide impairs the performance of a specialized parasitoid of an aphid host feeding on Brassica plants. Biol Control 66:49-55

Klaiber J, Najar-Rodriguez AJ, Piskorski R, Dorn S (2013b) Plant acclimation to elevated $\mathrm{CO}_{2}$ affects important plant functional traits, and concomitantly reduces plant colonization rates by an herbivorous insect. Planta 237:29-42

Kugimiya S, Shimoda T, Tabata J, Takabayashi J (2010) Present or past herbivory: a screening of volatiles released from Brassica rapa under caterpillar attacks as attractants for the solitary parasitoid, Cotesia vestalis. J Chem Ecol 36(6):620-628

Lawrence SD, Novak NG, Ju CJT, Cooke JEK (2008) Potato, Solanum tuberosum, defense against Colorado potato beetle, Leptinotarsa decemlineata (Say): microarray gene expression profiling of potato by Colorado potato beetle regurgitant treatment of wounded leaves. J Chem Ecol 34:1013-1025

Loughrin JH, Manukian A, Heath RR, Turlings TCJ, Tumlinson JH (1994) Diurnal cycle of emission of induced volatile terpenoids herbivore-injured cotton plants. Proc Natl Acad Sci USA 91(25):11836-11840

Mathur V, Tytgat TG, Hordijk CA, Harhangi HR, Jansen JJ, Reddy AS, Harvey JA (2013) An ecogenomic analysis of herbivore-induced plant volatiles in Brassica juncea. Mol Ecol 22:6179-6196

Mattiacci L, Rocca BA, Scascighini N, D'Alessandro M, Hern A, Dorn S (2001) Systemically induced plant volatiles emitted at the time of "danger". J Chem Ecol 27:2233-2252

Micha SG, Kistenmacher S, Molck G, Wyss U (2000) Tritrophic interactions between cereals, aphids and parasitoids: discrimination of different plant-host complexes by Aphidius rhopalosiphi (Hymenoptera: Aphidiidae). Eur J Entomol 97:539-543

Musser RO, Hum-Musser SM, Eichenseer H, Peiffer M, Ervin G, Murphy B, Felton GW (2002) Herbivory: caterpillar saliva beats plant defences. Nature 416:599-600

Najar-Rodriguez A, Orschel B, Dorn S (2013) Season-long volatile emissions from peach and pear trees in situ, overlapping profiles, and olfactory attraction of an oligophagous fruit moth in the laboratory. J Chem Ecol 39:418-429

Ngumbi E, Chen L, Fadamiro HY (2009) Comparative GC-EAD responses of a specialist (Microplitis croceipes) and a generalist (Cotesia marginiventris) parasitoid to cotton volatiles induced by two caterpillar species. J Chem Ecol 35:1009-1020
Pareja M, Qvarfordt E, Webster B, Mayon P, Pickett J, Birkett M, Glinwood R (2012) Herbivory by a phloem-feeding insect inhibits floral volatile production. PLoS One 7:e31971

Pike KS, Stary P, Miller T, Allison D, Graf G, Boydston L, Miller R, Gillespie R (1999) Host range and habitats of the aphid parasitoid Diaeretiella rapae (Hymenoptera: Aphidiidae) in Washington State. Environ Entomol 28:61-71

Piskorski R, Dorn S (2010) Early-season headspace volatiles from apple and their effect on the apple blossom weevil Anthonomus pomorum. Chem Biodivers 7:2254-2260

Scascighini N, Mattiacci L, D'Alessandro M, Hern A, Rott AS, Dorn $S$ (2005) New insights in analysing parasitoid attracting synomones: early volatile emission and use of stir bar sorptive extraction. Chemoecology 15:97-104

Schuman MC, Barthel K, Baldwin IT (2012) Herbivory-induced volatiles function as defence increasing fitness of the native plant Nicotiana attenuata in nature. eLife 1:e00007

Schwartzberg EG, Böröczky K, Tumlinson JH (2011) Pea aphids, Acyrthosiphon pisum, suppress induced plant volatiles in broad bean, Vicia faba. J Chem Ecol 37:1055-1062

Taveira M, Fernandes F, Guedes de Pinho P, Andrade PB, Pereira JA, Valentão $\mathrm{P}$ (2009) Evolution of Brassica rapa var. rapa L. volatile composition by HS-SPME and GC/IT-MS. Microchem J 93:140-146

Turlings TCJ, Wäckers F (2004) Recruitment of predators and parasitoids by herbivore-injured plants. In: Cardé RT, Millar J (eds) Advances in insect chemical ecology. Cambridge University Press, Cambridge, pp 21-75

Vallat A, Dorn S (2005) Changes in volatile emissions from apple trees and associated response of adult female codling moths over the fruit-growing season. J Agr Food Chem 53:4083-4090

Walling LL (2008) Avoiding effective defenses: strategies employed by phloem-feeding insects. Plant Physiol 146:859-866

Winde I, Wittstock U (2011) Insect herbivore counteradaptations to the plant glucosinolate-myrosinase system. Phytochem 72:15661575

Wittstock U, Kliebenstein DJ, Lambrix V, Reichelt M, Gershenzon J (2003) Glucosinolate hydrolysis and its impact on generalist and specialist insect herbivores. Recent Adv Phytochem 37:101-125

Zhang P-J, Xu C-X, Zhang J-M, Lu Y-B, Wei J-N, Liu Y-Q, David A, Boland W, Turlings TCJ (2013) Phloem-feeding whiteflies can fool their host plants, but not their parasitoids. Funct Ecol 27:1304-1312 